Title : will be set by the publisher Editors : will be set by the publisher EAS Publications Series, Vol. ?, 2009

e-mail: xfgong@niaot.ac.cn

\title{
DOME A SITE TESTING AND FUTURE PLANS
}

X. Gong ${ }^{2,1}$, L. Wang ${ }^{4,2,3}$, X. Cui ${ }^{1,2}$, L. Feng ${ }^{5}$, X. Yuan ${ }^{4,2}$, M. C. B. Ashley ${ }^{6}$, G. Allen ${ }^{7}$, C. S. Bonner ${ }^{6}$, S. G. Bradley ${ }^{8}$, J. R. Everett ${ }^{6}$, S. Hengst ${ }^{6}$, J. Hu ${ }^{4,2}$, Z. Jiang ${ }^{4,2}$, C. A. Kulesa ${ }^{9}$, J. S. Lawrence ${ }^{11,6,10}$, Y. Li ${ }^{12,2}$, D. M. Luong-Van ${ }^{6}$, M. J. McCaughrean ${ }^{13}$, A. M. Moore ${ }^{14}$, C. Pennypacker ${ }^{15}$, W. Qin ${ }^{12,2}$, R. Riddle ${ }^{16}$, Z. Shang ${ }^{17,2}$, J. W. V. Storey ${ }^{6}$, B. Sun ${ }^{12,2}$, N. Suntzeff ${ }^{3}$, N. F. H. Tothill ${ }^{13}$,

T. Travouillon ${ }^{16}$, C. K. Walker ${ }^{9}$, J. Yan ${ }^{5,2}$, H. Yang ${ }^{12,2}$, J. Yang ${ }^{5,2}$, D. G. York ${ }^{18}$, X. Zhang ${ }^{5,2}$, Z. Zhang ${ }^{12,2}$, X. Zhou ${ }^{4,2}$ and Z. Zhu ${ }^{5,2}$

\footnotetext{
${ }^{1}$ Nanjing Institute of Astronomical Optics and Technology, Nanjing 210042, China

2 Chinese Center for Antarctic Astronomy

${ }^{3}$ Physics Department, Texas A\&M University, College Station, TX 77843, USA

${ }^{4}$ National Astronomical Observatories, Chinese Academy of Sciences, Beijing 100012, China

${ }^{5}$ Purple Mountain Observatory, Nanjing 210008, China

${ }^{6}$ School of Physics, University of New South Wales, NSW 2052, Australia

7 Solar Mobility Pty Ltd, Thornleigh, NSW 2120, Australia

${ }^{8}$ Physics Department, University of Auckland, Auckland 1142, New Zealand

${ }^{9}$ Steward Observatory, University of Arizona, Tucson, AZ 85721, USA

10 Department of Physics and Engineering, Macquarie University, NSW 2109, Australia

11 Anglo-Australian Observatory, NSW 1710, Australia

12 Polar Research Institute of China, Pudong, Shanghai 200136, China

13 School of Physics, University of Exeter, Exeter, EX4 4QL, UK

14 Caltech Optical Observatories, California Institute of Technology, Pasadena, CA 91125, USA

${ }^{15}$ Lawrence Berkeley National Laboratory, University of California, Berkeley, CA 94720, USA

16 Thirty Meter Telescope Project, Pasadena, CA 91107, USA

17 Tianjin Normal University, Tianjin 300074, China

18 Department of Astronomy and Astrophysics and The Enrico Fermi Institute, University of Chicago, Chicago, IL 60637, USA
} 


\begin{abstract}
In January 2005, members of a Chinese expedition team were the first humans to visit Dome A on the Antarctic plateau, a site predicted to be one of the very best astronomical sites on earth.

In 2006, the Chinese Center for Antarctic Astronomy (CCAA) was founded to promote the development of astronomy in Antarctica, especially at Dome A. CCAA has since taken part in two traverses to Dome A, organized by the Polar Research Institute of China (PRIC), in the austral summers of $2007 / 2008$ and $2008 / 2009$. These traverses resulted in the installation of many site-testing and science instruments, supported by the PLATO observatory. The Chinese Small Telescope ARray (CSTAR) has produced excellent results from Dome A.

Our future plans include further site-testing work, and the following full-scale science instruments: three 0.5-m Antarctic Schmidt Telescopes (AST3), and a proposed 4-m telescope for wide-field infrared high spatial-resolution surveys. The first AST3 telescope is under construction and is scheduled for installation in 2011.
\end{abstract}

Keywords: Dome A, CCAA, site testing, PLATO, CSTAR, AST3

\title{
1 Introduction
}

For modern, large, astronomical telescopes, site selection is of crucial importance. The high plateau of Antarctica has proven characteristics that make it almost certain to contain the best astronomical observing sites on earth. For example, Dome $\mathrm{C}$ (3260m altitude) on the plateau shows a median seeing of 0.27 arcseconds above a 30m turbulent boundary layer (Lawrence et al. 2004), and below 0.15 arcseconds for $25 \%$ of the time. Dome A (4093m altitude), the highest point on the plateau, may be expected to be an even better astronomical site. Figure 1 shows the positions of these sites within Antarctica. Apart from the superb seeing conditions, the Antarctic plateau offers the following advantages: long periods of continuous observation (more than 4 months for Dome A in winter), which is an advantage for many projects, e.g., astroseismology, and the detection of transiting planets; low wind-speed; low scattered light and high atmospheric transparency; very cold and dry atmosphere; low infrared background; low scintillation; a wide isoplanatic angle and a long coherence time; and low seismic activity. All these advantages make the Antarctic plateau the most attractive site on earth for optical/infrared astronomy (see, e.g., Ashley et al. 2004), and almost certainly the single best site for submillimeter and terahertz astronomy.

In January 2005, a Chinese expedition team was the first to reach Dome A, and the Chinese Government decided to establish a scientific station there during the International Polar Year in 2009. This provided an exciting opportunity for the Chinese astronomical community and their international collaborators to start the site-testing and astronomical observation at Dome A. The Chinese Center for Antarctic Astronomy (CCAA) was founded during 2006 with members from several astronomical institutes, observatories and universities within China. The 
CCAA, with support from the Chinese Academy of Sciences (CAS) and National Science Foundation of China (NSFC), has since coordinated astronomical research work at Dome A during 2008 and 2009. Through a large international collaboration, the PLATO (PLATeau Observatory) and Chinese first Antarctic telescope CSTAR (Chinese Small Telescope ARray) were installed at Dome A at January 2008, and operated unattended for 7 months from January to August. CSTAR consists of four 14.5-cm wide-field telescopes for astronomical observation and sitetesting at Dome A (Yuan et al. 2008, Cui et al. 2008). CSTAR is mainly designed for the detection of variable stars, and the measurement of atmospheric extinction, sky background and cloud coverage. Following repair and maintenance in January 2009, PLATO and CSTAR worked again throughout the 2009 winter. Preliminary data are very promising for the potential of the Dome A site for astronomy (Yang et al. 2009).

\section{Dome A site testing campaign: 2008 and 2009}

Dome $\mathrm{A}$ is $4093 \mathrm{~m}$ above sea-level, and located at $80^{\circ} 22^{\prime} 00^{\prime \prime} \mathrm{S}, 77^{\circ} 21^{\prime} 11^{\prime \prime} \mathrm{E}$, which is the highest location on the Antarctic plateau. Since as yet there is no winterover capability at Dome A, our collaborators at the University of New South Wales (UNSW) designed and built a facility called PLATO ("PLATeau Observatory", Lawrence et al. 2009, Hengst et al. 2008, Ashley et al. 2009) to provide power, heat, computer control and satellite communication for our experiments. PLATO and its first generation of instruments were installed by the second expedition to Dome A - the 24th Chinese Antarctic expedition team, which included two astronomers from the Chinese Center for Antarctic Astronomy: Xu Zhou and Zhengxi Zhu. Apart from CSTAR, other instruments provided by the international collaboration include:

- Gattini: two optical cameras determining the optical sky brightness and cloud cover statistics,

- DASLE: an array of sonic anemometers measuring the meteorological conditions within the near surface layer,

- Pre-HEAT: a submillimeter tipping radiometer measuring the atmospheric transmission and water vapor content, and able to image the Galactic Plane in spectral lines (Kulesa et al. 2008, Walker et al. 2004), and

- Snodar: an acoustic radar designed to measure turbulence in the atmosphere in the surface layer, up to about 200m (Bonner et al. 2009).

PLATO and its instruments ran unattended for 204 days during 2008 until the system was turned off remotely from UNSW due to an exhaust pipe failure in the Engine Module. Highlights of the scientific results from 2008 include: the PreHEAT instrument showing that Dome A is likely to be the driest location on the surface of the planet, opening up new windows for terahertz astronomy; the Snodar 
instrument proving a novel instrument concept, and confirming expectations of a low turbulent boundary layer height; and CSTAR has acquired about 360G data, which is now being analysed for astronomical phenomenon such as variable stars, binaries, and possible transiting planets.

During the summer of 2008/9, the 25th Chinese Antarctic expedition team, including one of us, Xuefei Gong, made its way to Dome A. The main objective of the traverse was to begin construction of the new summer station, named Kunlun. The expedition arrived at Dome A on January 6, 2009 with 570 tonnes of cargo after a hard 24-day journey from the Chinese Antarctic coastal station Zhongshan. The team spent 27 days at Dome A and finished the construction of Kunlun station. Simultaneously, with the help of many team members, PLATO was refurbished and new astronomical instruments were installed as follows:

- Upgrade and maintain PLATO, including the Engine and Instrument Modules,

- Install a new Snodar and repair the original Snodar,

- Install a new Gattini camera and Nigel spectrometer,

- Swap control computers for the CSTAR telescopes,

- Install five wireless web-cameras, and

- Collect vibration and shock data from Fremantle to Dome A, to aid in the future transportation of precision instruments.

At the time of writing (2009 August 21), PLATO has been running for 221 days during 2009. All the site-testing and scientific instruments have worked well. All of them observed throughout the Antarctic winter and collected site-testing data, reduced versions of which have been uploaded from Antarctica using Iridium modems.

Figure 2 show the experiments at Dome A as of January 2009, including PLATO's Engine Module, Instrument Module, and the following instruments: CSTAR, DASLE and two Snodars. Gattini and Pre-HEAT are mounted on the Instrument Module.

\section{Kunlun Station and future plans for Dome A}

Apart from its astronomical advantages, Dome A is an ideal site for studying global climatic and environmental changes. It is one of the most suitable sites for drilling to obtain deep ice cores with an age exceeding one million years. It is also a favorable site for monitoring and detecting global background atmospheric baselines. As a part of the "PANDA Plan", a core component of the International Polar Year China Action, the Chinese government selected the Dome A site for China's first inland station. From 2009 January 7-27, the 25th Chinese Antarctic Expedition inland team constructed the first part of the new Kunlun Station, 
Figure 3. Following completion of the second part of the station, scheduled for 2010 , the total constructed area will be $620 \mathrm{~m}^{2}, 216 \mathrm{~m}^{2}$ of which is a containertype building, and $404 \mathrm{~m}^{2}$ for the assembly building. The short term objective is to accommodate 15-20 people over summer, with a long term objective to provide for 25 people living year-round.

The construction and operation of Kunlun Station will provide infrastructure support for the entire Dome A scientific program, which includes a large astronomy component. The Chinese Center for Antarctic Astronomy plans to deploy additional site-testing instruments, and large-scale astronomical telescopes at Dome A through broad international cooperation. In the near future the possible instruments include:

- lunar SHABAR (Shadow Band Ranger), an array of photodiodes which measures the variation of lunar intensity to determine the profile of nighttime surface-layer turbulence,

- MASS (Multi-Aperture Scintillation Sensor), an optical scintillometer which measures the atmospheric turbulence profile in the earth's atmosphere from $0.5 \mathrm{~km}$ to $20 \mathrm{~km}$ in altitude, with low spatial resolution and high temporal resolution,

- FTS (Fourier Transform Spectrometer), broadband measurement of atmospheric emission in the submillimeter,

- AST3 (Antarctic Schmidt Telescope array), an array of three 0.5-m wide-field optical Schmidt telescopes designed to obtain light curves for a large sample of Type Ia supernovae and to search for exoplanets through gravitational microlensing, and

- DIMM (a automated differential image motion monitor telescope), measuring the integrated seeing at Dome A.

At the same time, the Chinese Center for Antarctic Astronomy is proposing several large-scale scientific facilities for the longer term:

- 2-m LAMOST - South LAMOST project, complementing the 4-m LAMOST at Xinglong Station for a full-sky spectroscopic survey,

- a 4-m Antarctic optical/IR telescope, a 3D model of which is shown in Figure 4 , and

- a 15-m sub-millimeter/THz telescope.

\section{Conclusion}

We have successfully operated site-testing and science instruments over two winters at Dome A, with no humans on site. The results are revealing the potential for Dome A to be an excellent location for an astronomical observatory. The 
Chinese Center for Antarctic Astronomy is dedicated to long term site-testing research with international collaborators, and also plans to construct large-scale scientific facilities for astronomical observations, eventually realizing the dream of an Antarctic astronomical observatory at Dome A.

\section{References}

Ashley, M. C. B., et al. 2004, Astron. Nachr, No. 6-8, 619-625

Ashley, M. C. B., et al. 2009, this volume

Bonner, C. S., et al. 2009, Acoustics Australia, 37, 47-51

Cui, X., Yuan, X., \& Gong, X. 2008, Proc. SPIE, 7012,70122D

Hengst, S., Allen, G. R., Ashley, M. C. B., Everett, J. R., Lawrence, J. S., Luong-Van, D., \& Storey, J. W. V. 2008, Proc. SPIE, 7012,70124E

Kulesa, C. A. et al. 2008, SPIE, 7012, 701249-1-701249-11

Lawrence, J. S., Ashley, M. C. B., Tokovinin, A., \& Travouillon, T. 2004, Nature, 431, $278-281$

Lawrence, J. S., Ashley, M. C. B., Hengst, S., Luong-Van, D. M., Storey, J. W. V., Yang, H., Zhou, X. \& Zhu, Z. 2009, Rev. Sci. Inst., 80, 064501-1-064501-10

Walker, C. K., et al. 2004, Proc. SPIE, 5489, 470

Yang, H., et al. 2009, PASP, 121, 174-184

Yuan, X., et al. 2008, Proc. SPIE, 7012,70124G 


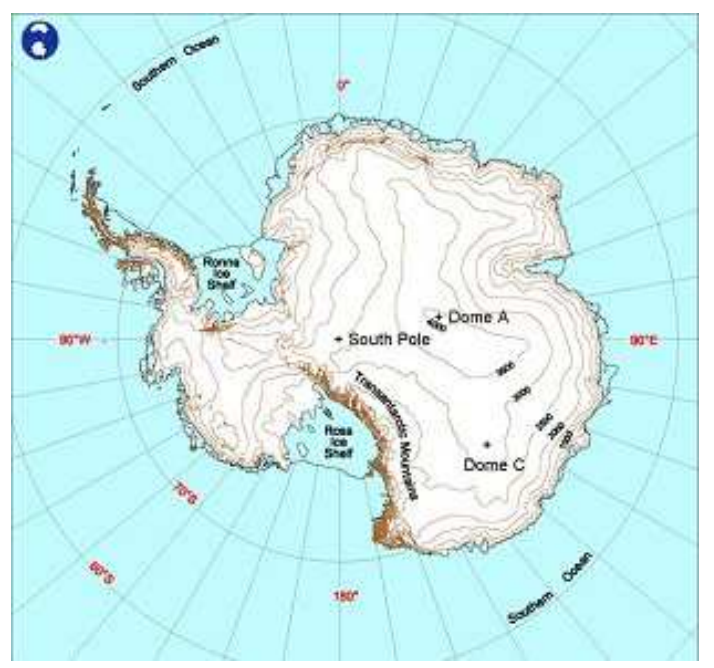

Fig. 1. A map of Antarctica, showing the inland stations.

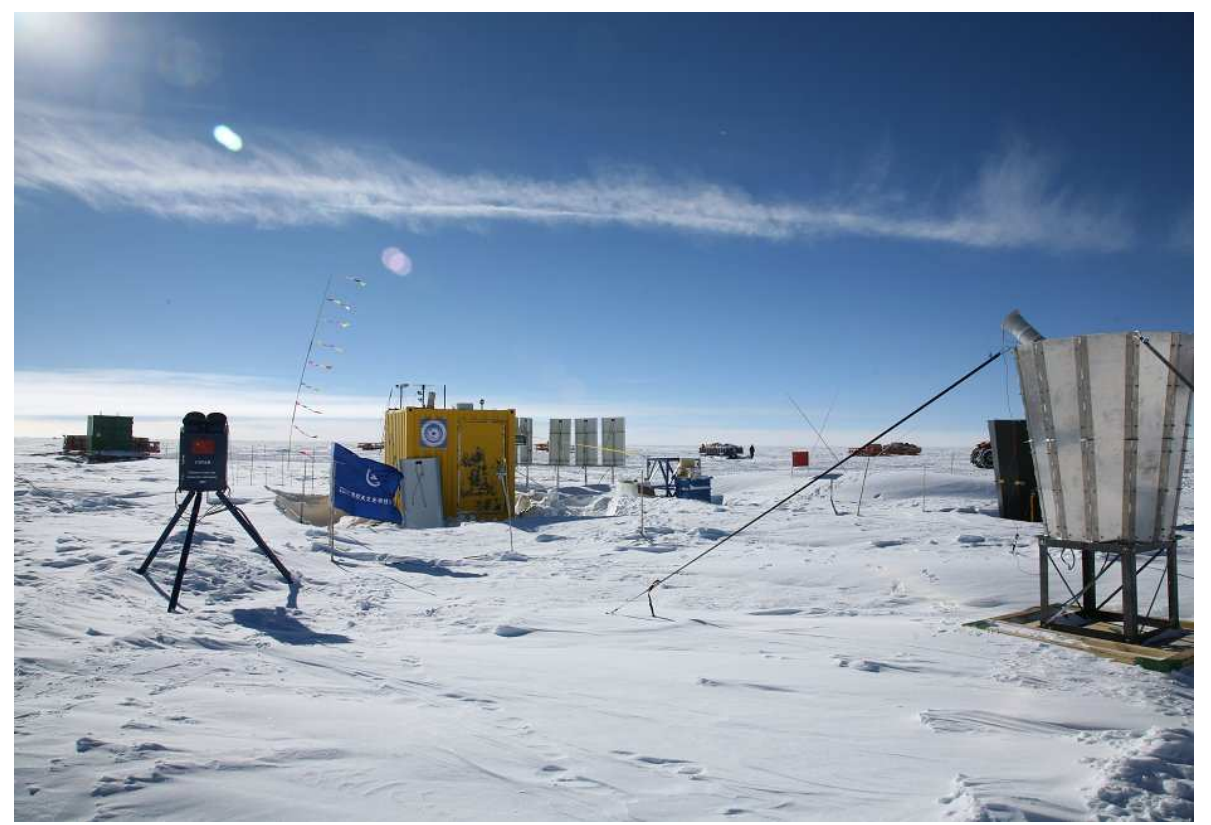

Fig. 2. The astronomical facilities at Dome A, January 2009. 


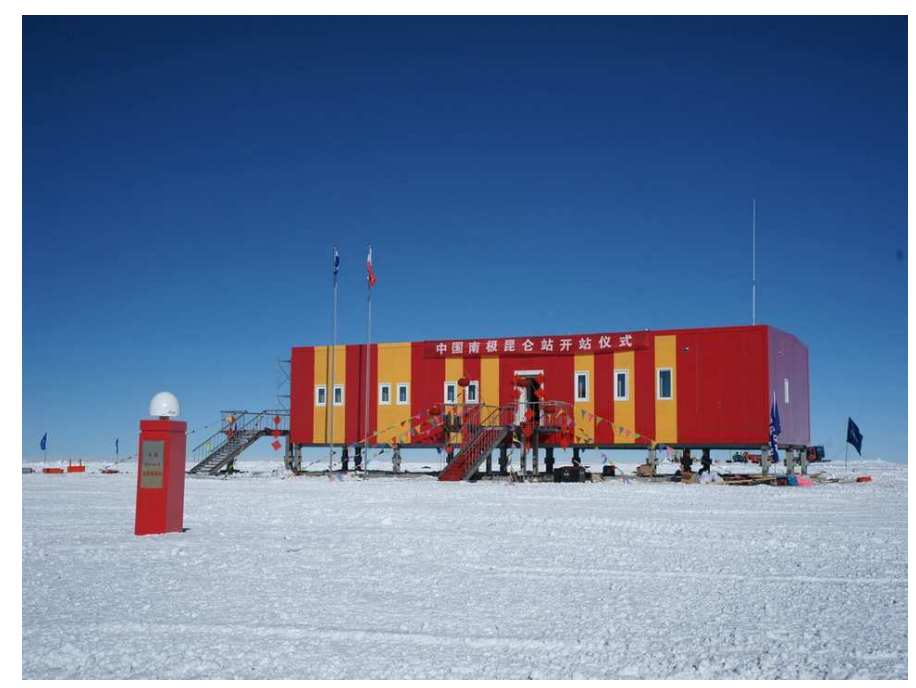

Fig. 3. The Chinese Kunlun Station.

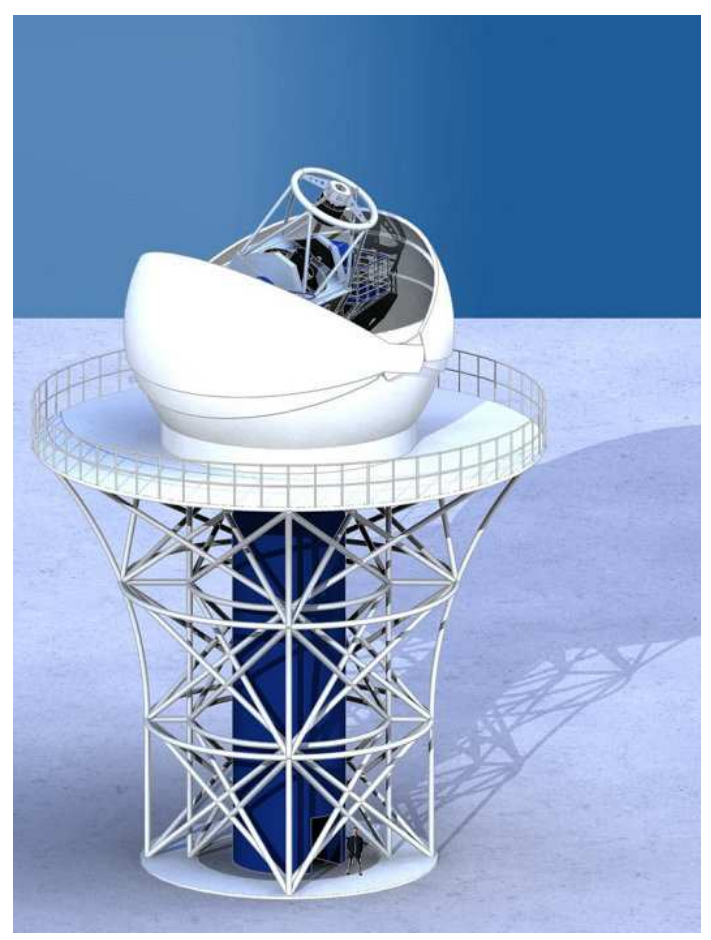

Fig. 4. The proposed 4-m Antarctic Telescope. 